\title{
Role of Neuronal Synchronizing Mechanisms in the Propagation of Spreading Depression in the in vivo Hippocampus
}

\author{
Oscar Herreras, ${ }^{1}$ Carlota Largo, ${ }^{1}$ José M. Ibarz,, George G. Somjen, ${ }^{2}$ and Rafael Martín del Río' \\ 'Departamento Investigación, Hospital Ramón y Cajal, 28034 Madrid, Spain and ²Department of Cell Biology, Duke \\ University Medical Center, Durham, North Carolina 27710
}

To detect what initiates spreading depression (SD), the early prodromal events were investigated in hippocampal CA1 of urethane-anesthetized rats. SD was provoked by microdialysis or focal microinjection of high-K+ solution. Extracellular DC potentials and extracellular potassium concentration $\left(\left[\mathrm{K}^{+}\right]_{0}\right)$ were recorded, and spontaneous and evoked potentials analyzed for current source-density (CSD). In the front of an approaching SD wave, several seconds before the onset of the typical sustained negative potential shift $\left(\Delta \boldsymbol{V}_{0}\right)$ and the increased $\left[\mathrm{K}^{+}\right]_{0}$, fast electrical activity was detected. This consisted initially of small rhythmic (60-70 $\mathrm{Hz}$ ) sawtooth wavelets, which then gave way to a shower of population spikes (PSs) of identical frequency. Prodromal wavelets and PSs were synchronized over considerable distances in the tissue. Sawtooth wavelets were identified as pacemakers of the prodromal PS burst. Simultaneous recording at three depths revealed that the spontaneous prodromal PSs occurred exactly in phase in dendrites and somata whereas synaptically transmitted PSs arose first in the proximal dendrites and were conducted from there into the soma membrane. During a spike burst, stratum (st.) pyramidale served as current sink, while in the proximal sublayer of st. radiatum spike-sinks gave way to spike sources that grew larger as the sinks in st. pyramidale began to subside. Blocking synaptic transmission did not abolish the prodromal spike burst, yet repetitive orthodromic activation inhibited it without altering the subsequent SD waveform. Complex changes in cell excitability were detected even before fast spontaneous activities. We concluded that, in the initial evolution of SD, changes in neuron function precede the regenerating depolarization by several seconds. We propose that the opening of normally closed electric junctions among neurons can best explain the long-distance synchronization and the flow current that occurs in the leading edge of a propagating wave of SD.

IKey words: spreading depression, CA1, neuronal synchronization, current source-density, microdialysis, in vivo, evoked potentials, cell-to-cell communication, fast rhythmic activity]

Received Feb. 25, 1993; revised May 9, 1994; accepted May 16, 1994

This work has been supported by Grant $93 / 661$ from the Fondo de Investigaciones Sanitarias of Spain. Some of the experiments were performed at Duke University and were supported by Grant NS 18670 from the NIH. We thank Dr. L. Barrio for the critical reading of the manuscript.

Correspondence should be addressed to Dr. O. Herreras, Departamento Investigación. Hospital Ramón y Cajal, Ctra. Colmenar km 9, Madrid 28034, Spain.

Copyright (C) 1994 Society for Neuroscience $0270-6474 / 94 / 147087-12 \$ 05.00 / 0$
Half a century after its discovery (Leão, 1944), the physiological changes responsible for the generation and propagation of Leão's spreading depression (SD) remain obscure. Stimuli triggering $\mathrm{SD}$, ionic disturbances of the extracellular milieu, and relation to neuronal function impairment are well known (for review, see Marshall, 1959; Bureš et al., 1974; Nicholson, 1984), but its biophysical basis is far from clear.

Widely accepted hypotheses hold that the primary event responsible for both the initiation and the propagation of SD is the release of some substance from neuronal elements to the extracellular compartment, which initially excites and then depresses adjacent neurons. The slowness of diffusion of the mediator would account for the low velocity of SD propagation. Among the substances proposed to mediate SD propagation are potassium (Grafstein, 1956; Brinley et al., 1960; Bureš and Křivánek, 1960) and excitatory amino acids (Van Harreveld, 1959; Siesjö and Bengtsson, 1989; Fabricius et al., 1993). There are, however, observations that are difficult to reconcile with either of these two propositions (Lehmenkïhler, 1990; Herreras and Somjen, 1993a,b; see also Discussion).

$\mathrm{SD}$ had been interpreted as a composite process or a sequence of several linked events. To solve its genesis, a most important question concerns identification of the very first step in the chain reaction. In the extant literature, however, generally more attention has been given to the major depolarization and the attending extracellular potential shift $\left(\Delta V_{o}\right)$ than to the antecedent events. Among antecedents heralding the onset of SD that have bcen reported, are a slight increase of extracellular potassium $\left(\left[\mathrm{K}^{+}\right]_{o}\right)$, a small positive shift preceding the fast negative shift of the extracellular potential $\left(\Delta V_{o}\right)$ (Marshall, 1959), and several types of fast field activity including a short burst of action potentials or intense synaptic noise (Leão, 1944; Grafstein 1956; Rosenblueth and García-Ramos, 1966; Ichijo and Ochs, 1970; Higashida et al., 1974; Somjen and Aitken, 1984; Haglund and Schwartzkroin, 1990; Herreras and Somjen, 1993a). Even a silence of spontaneous or evoked activity has occasionally been described prior to other signs (Grafstein, 1956; Morlock et al., 1964; Muñoz, 1970; Higashida et al., 1974). Not all of these early signs are obligatory prodromals of the large, accelerating, regenerative depolarization that is typical of the process.

The purpose of this article is to examine the earliest events that lead to SD. We chose the hippocampus for this investigation, because its simple and regular cytoarchitecture facilitates interpretation of electrical recordings. Prcviously, we have presented evidence that waves of SD can propagate independently among somata and dendrites of the same cells (Herreras and Somjen, 1993a,d). We have also defined different components 
of the SD wave that behave differently, have distinct associated ionic shifts and differential sensitivity to Glu receptor blockers (Herreras and Somjen, 1993a,b,d). By simultaneous recordings with multiple microelectrodes, current source-density (CSD) analysis of field potentials, ion-selective microelectrodes, and microdialysis, we have now undertaken a detailed spatiotemporal analysis of the earliest noticeable spontaneous or evoked electrophysiological manifestations associated to the SD reaction. We are concluding that, at least in the CA1 sector, SD propagation is mediated by neither potassium ions nor Glu receptor activation. Our findings are compatible with the hypothesis put forward earlier, suggesting that the opening of electrotonic junctions among neurons is a significant early event in the evolution of SD (Somjen et al., 1992).

Some of the data described here have been presented in preliminary form at a meeting (Largo et al., 1993).

\section{Materials and Methods}

Preparation and recording. Female Sprague-Dawley rats (39 animals, $225-250 \mathrm{gm}$ ) were anesthetized with urethane $(1.2-1.5 \mathrm{gm} / \mathrm{kg}$, i.p.). The animals were breathing spontaneously. Body temperature was maintained at $37 \pm 0.1^{\circ} \mathrm{C}$ with a heating blanket. Surgery and stereotaxic procedures were carried out as previously described in detail (Herreras, 1990). Concentric bipolar stimulating electrodes were placed at one or the two of the following locations: CA3 sector for activation of the ipsilateral Schaffer collaterals (from bregma, midline, and cortical surface, A - 3.2, L 2.6, V -3.3); alveus for antidromic activation (A - 5.5, $\mathrm{L} 2.6, \mathrm{~V}-1.8)$. These points were approached at a $30^{\circ}$ angle in the sagittal plane, and depth was subsequently adjusted after placement of the recording electrodes to find the point of lowest threshold for the desired evoked response.

Recording electrodes were glass micropipettes filled with $150 \mathrm{~mm}$ $\mathrm{NaCl}$ (5-10 MR). Micropipettes were sometimes used in vertical arrays of two or three pipettes glued so as to record from different depths at essentially the same AP and L coordinates. These arrays were constructed and used as previously described (I Ierreras and Somjen, 1993b).

Double barreled ion-selective microelectrodes were manufactured as described elsewhere (Somjen, 1981). The Fluka ion exchanger for $\mathrm{K}^{+}$ (no. 60031) was used as liquid membrane. Electrodes were calibrated before and after each experiment and results were rejected on rare occasion when the two differed by more than $10 \%$. The liquid membrane was approximately $800 \mu \mathrm{m}$ long. The reference barrel served for the recording of evoked potentials and DC voltages. The voltages recorded by the ion-selective electrodes were transformed to a linear millimolar scale with the help of the AXUM program, taking into account the calibrated sensitivity of each electrode.

The characteristic configuration of evoked potentials guided the placement of the recording electrodes (Herreras, 1990). In previous studies (Herreras et al., 1987) electrolytic lesions and dye marks were made to verify post-mortem the placement of stimulating and recording electrodes. Histology invariably verified the reliability of electrophysiological criteria.

The $\mathrm{Ag} / \mathrm{AgCl}$ wire of recording micropipette electrodes was connected to DC-coupled FET input stages. A subcutaneous $\mathrm{Ag} / \mathrm{AgCl}$ wire electrode served as reference. After amplification, DC signals were recorded on VCR and processed by AXOTAPE computer program either at 5-20 $\mathrm{Hz}$ or at $5-10 \mathrm{kHz}$ sample rate and then further analyzed by the AXUM program. When necessary, signals were reacquired in the computer after AC coupling ( $1 \mathrm{~Hz}$ to $5 \mathrm{kHz}$ ). In some cases, noisy potentials were smoothed by filtering using the Savitsky-Golay algorithm described in the AXUM manual. Given the small amplitude and characteristic frequency of some of the signals of interest in this study $(<1 \mathrm{mV}, 60-70$ $\mathrm{Hz}$ ) it was often necessary to minimize $50 \mathrm{~Hz}$ line noise. This was made off line by algebraic substraction of an averaged portion of $50 \mathrm{~Hz}$ noise from the row signal after careful time reset of the noise. A similar substraction procedure was employed to filter out large-amplitude EEG waves from small portions of signal for the sake of figure presentation.

Microdialysis. Microdialysis was used to perfuse drugs into the tissue and to elicit SD waves by perfusion of high-potassium solutions. Dialysis probes were manufactured as previously described (Herreras and Somjen, 1993a), except that an inner silica cannula (140 $\mu \mathrm{m}$ o.d., $40 \mu \mathrm{m}$ i.d.) was employed instead of the stainless steel cannula. The active dialysis membrane was a cylinder $800 \mu \mathrm{m}$ long of $220 \mu \mathrm{m}$ o.d.

Before inserting the probe in the brain a micropipette electrode was first introduced to find an appropriate location, guided by recording the evoked potential. The probe was lowered thereafter at the same position so that the entire dorsoventral extension of the CAl region was exposed to dialysis. The usual location was A -4.5 to $-5, \mathrm{~L} 2.6$, but sometimes a more posterior and lateral position was aimed to allow maximal room for anteriorly placed recording electrodes. Just before the probe reached its final position an SD wave was invariably recorded at a near rostral micropipette due to the mechanical trauma caused during the probe implantation.

A control artificial cerebrospinal fluid (ACSF) of the following composition was initially perfused (in mmol/liter): $122 \mathrm{NaCl}, 3 \mathrm{KCl}, 0.4$ $\mathrm{KH}_{2} \mathrm{PO}_{4}, 1.2 \mathrm{CaCl}_{2}, 1.2 \mathrm{MgSO}_{4}, 25 \mathrm{NaHCO}_{3}$. High-potassium solution was prepared by replacing $100 \mathrm{mmol} /$ liter $\mathrm{NaCl}$ by $\mathrm{KCl}$. All drugs perfused were dissolved in ACSF or high- $\mathrm{K}^{+}$solutions. The dialysis fiber was perfused by means of a Gilson peristaltic pump at a constant rate of $2 \mu \mathrm{l} / \mathrm{min}$. A $90 \mathrm{~min}$ rest period was allowed after the probe implantation before the experiment began.

Single SD waves were elicited by 5-8 min pulses of high- $\mathrm{K}^{+}$perfusion through the dialysis probe. A $15 \mathrm{~min}$ rest period was used between consecutive SD episodes. Continuous high- $\mathrm{K}^{+}$perfusion generated an SD focus from which waves of SD emanated at intervals increasing from about 5 to about $10 \mathrm{~min}$ (Herreras and Somjen, 1993a,b,d). When the effect of a drug on SD was studicd, a 15-30 min pcriod was allowed for the drug to diffuse out of the dialysis fiber and to achieve a steady gradient before the high- $\mathrm{K}^{+}$pulse. The actual concentration of drugs at specific distances from the dialysis fiber cannot be determined. From our experience, the concentration of hydrosoluble compounds that produces a definite electrophysiogical effect at a distance of 300-500 $\mu \mathrm{m}$ away from the fiber must be about 10 times higher in the perfusate than that required to exert the same effect as when administered in the bathing fluid of in vitro preparations (Herreras et al., 1989; Herreras and Somjen, 1993a,b).

The glutamate receptor blocking drugs used in this study, 6,7-dinitroquinoxaline-2,3-dione (DNQX) and 3-(( \pm$)$-2-carboxypiperazine-4yl)-propyl-1-phosphonic acid (CPP), were obtained from Tocris Neuramin (Essex, England), and all ACSF constituents and other inorganic ions, from Sigma (St. Louis).

Pressure ejection of high- $K^{+}$solution. In other experiments, SD waves were provoked by pressure ejection of $\mathrm{KCl}(1 \mathrm{M})$ from a glass micropipette (2-5 $\mu \mathrm{m}$ at the tip). The same pipette served for electrical monitoring of evoked potentials during implantation and subsequent registration of the voltage at the site of the SD initiation. Pressure was chosen so that microdrops of $25-50 \mathrm{nl}$ were produced outside the brain. When no pressure was applied, a stable DC baseline and normal evoked potentials recorded from the high- $\mathrm{K}^{+}$pipette indicated that leak of $\mathrm{K}^{+}$ was readily buffered by surrounding cells. Equal volumes of $1 \mathrm{M} \mathrm{NaCl}$ ejected from similar pipettes did not noticeably disturb evoked potentials recorded a few seconds later, indicating that the ejected volume did not disturb the neurons.

Current source-density (CSD) analysis and excitability study. CSD analysis permits the registration of "sources" wherc clectric current enters, and "sinks" where current leaves the interstitial space in a tissue. We used the customary unidimensional approximation of CSD (Stone and Freeman 1971; Freeman and Nicholson, 1975; Herreras, 1990; Herreras and Somjen, 1993c). A detailed account of technical and theoretical considerations has been presented elsewhere (Herreras, 1990).

In the present experiments, instead of plotting the depth profiles of regularly repeated field potentials, using a single moving pipette, a triple micropipette assembly was fashioned that permitted the simultaneous recording at three positions in the same vertical track. This procedure was required because of the variability of the fast potentials at the onset of the SD. The assembly was lowered at successive steps, each of them recording a single SD episode. The vertical separation between the tips was $100-125 \mu \mathrm{m}$ in different arrays. CSD was calculated at each station for the central one of the three electrode tips. Thesc distances arc larger than optimal (Herreras, 1990), but allowed for a nearly parallel orientation of the pipettes and therefore less tissue damage. On-line CSD computation of orthodromic evoked potentials served to control for tissue distortion and to ensure that the electrode assembly did not introduce artifactual currents. Otherwise, arrays were rejected. CSD of fast transients was calculated from the AC-coupled signals. Since the 

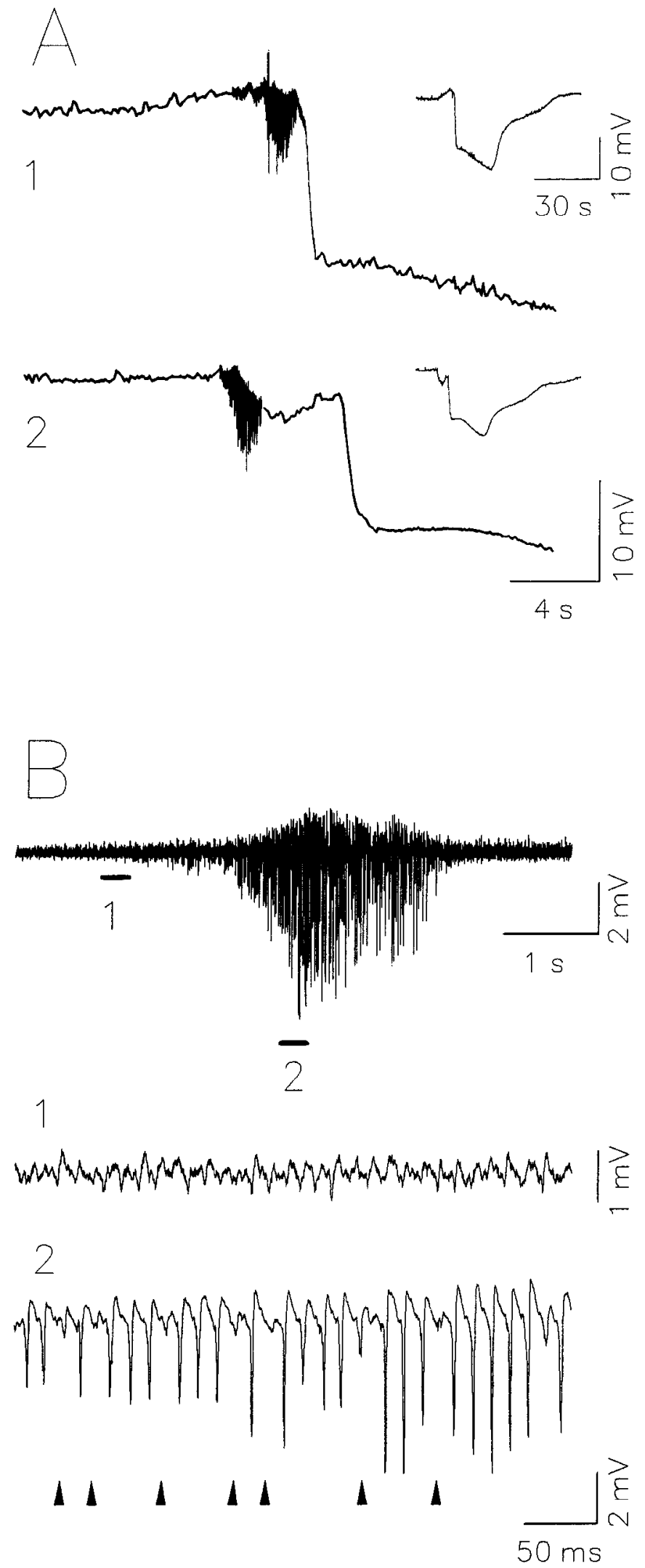

Figure 1. Features of the prodromal PS burst. $A$, DC-coupled recordings in the stratum (st.) pyramidale of two rats. The initial moments of SD waves, showing the PS burst and initial $\Delta V_{o}$ shift. The insets show the complete waveform of the $\Delta V_{\mathrm{o}}$ shift. $B$, Detail of an AC-coupled record of a PS burst in st. pyramidale. Below, traces labeled $l$ and 2 show expanded portions of the upper trace. Arrowheads mark failures tissue resistivity was unknown, only the second spatial derivative of the field potential gradient was calculated and therefore we used arbitrary proportional units (Herreras, 1990; Herreras and Somjen, 1993b)

\section{Results}

\section{Early activity}

Whenever an SD wave was elicited in the hippocampal CA1 region by any method, the sustained $\Delta V_{o}$ shift was preceded and its early phase was accompanied by a characteristic sequence of fast focal electric activities. Initially, there appeared small sawtooth-shaped potentials, which then gave way to a shower of population spikes (PSs). The amplitude of the spikes first increased and then decreased giving the burst a triangular-shaped envelope (Fig. $1 B$ ). After subsidence of the prodromal spike burst, the sawtooth wavelets often became again visible.

When recording from the cell body layer, the prodromal burst of PSs began to develop a few seconds before the characteristic $\Delta V_{o}$ shift, it lasted $2-4 \mathrm{sec}$, and it was already exhausted by the time the fast DC drop began at this layer (Fig. 1). Sawtooth wavelets were detectable preceding the PS burst by $2-3 \mathrm{sec}$. Most often the burst set out from a flat DC level or during the small positive hump that sometimes preceded the negative $\Delta V_{o}$ (Fig. 1 $A 1$ ). Occasionally, the spike burst was partially riding on an initial negative DC excursion (Fig. 1 $A 2$ ). The maximal amplitude of the PSs within a prodromal burst was $4-10 \mathrm{mV}$, always smaller than the maximal orthodromically evoked PS that ranged from 12 to $15 \mathrm{mV}$. The frequency of the spikes varied little in any one experiment. The mean interspike interval was 14.7 , with SEM of $\pm 0.12 \mathrm{msec}$ ( $n=82$, in 36 animals), corresponding to a frequency of $68 \mathrm{~Hz}$ (range, $43-89 \mathrm{~Hz}$ ). In a few (three animals) exceptional cases only unusually small PSs were seen at intervals that were two to three times greater than usual; these were not included in the statistics just presented.

The small sawtooth waves that preceded the spike burst had the same frequency as the spike burst itself. Occasionally a PS failed within the burst. In place of the missing PS a sawtooth wavelet was invariably recorded, and the next PS occurred at the expected time (Fig. $1 B 2$, arrowheads) without resetting the rhythm.

To examine temporal relations, two electrodes were placed at the same AP and L coordinates, one in st. pyramidale and the other in st. radiatum, as well as a third electrode in st. pyramidale $1 \mathrm{~mm}$ anterior to the other two (Fig. $2 A$ ). Remarkably, the fast activity recorded at the three sites (Fig. 2C,D) was precisely phase locked even when the different sites were experiencing different phases of the propagating SD wave. In the example of Figure 2 the spike burst at the caudal recording site (electrodes 2 and 3) that was near the origin of the SD wave was already declining whereas at the more distant anterior site (electrode 1) the pacemaker sawtooth activity was just beginning. Same temporal relationships were obtained in five more experiments using multiple recordings.

\section{The pattern of PS bursts in different cytoarchitectonic layers}

Triple electrodes were fastened so that their tips lay at different depths but very nearly at the same AP and L coordinates. Figure 3 illustrates the SD waves recorded with such assemblies at

of PSs within the burst. Negative potential is shown downward in all figures. 

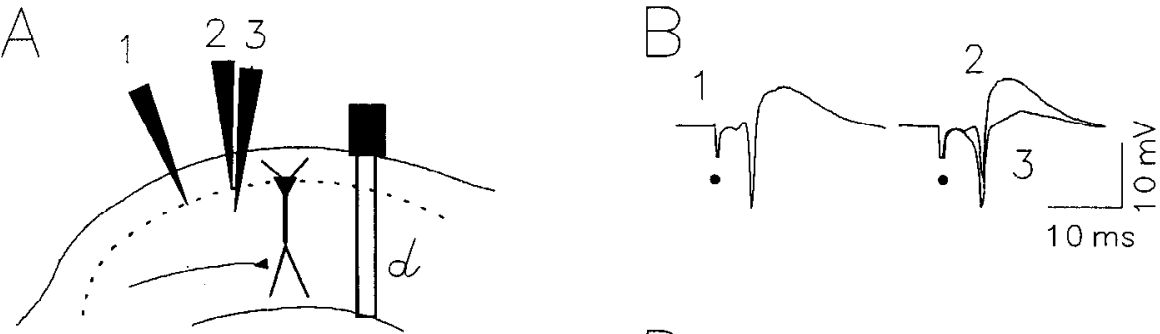

Figure 2. Phase relationship of fast activities. $A$, Scheme of electrode arrangement. Electrodes $I$ and 2 are located in st. pyramidale separated by 1 $\mathrm{mm}$ in anteroposterior direction; electrode 3 is located in proximal st. radiatum, $90 \mu \mathrm{m}$ below $2 . d$ indicates the position of the dialysis fiber (not to scale). $B$, Control evoked potentials to an orthodromic shock delivered in CA3. $C$, DC-coupled simultaneous recordings from three sites. $D$, Expanded portions of traces from $C$, marked by solid dots and labeled $a-c$, illustrating phase locking of fast activities. Note that all three recordings from site $I$ illustrated in $C$ were taken before the PS burst arrived to this location.
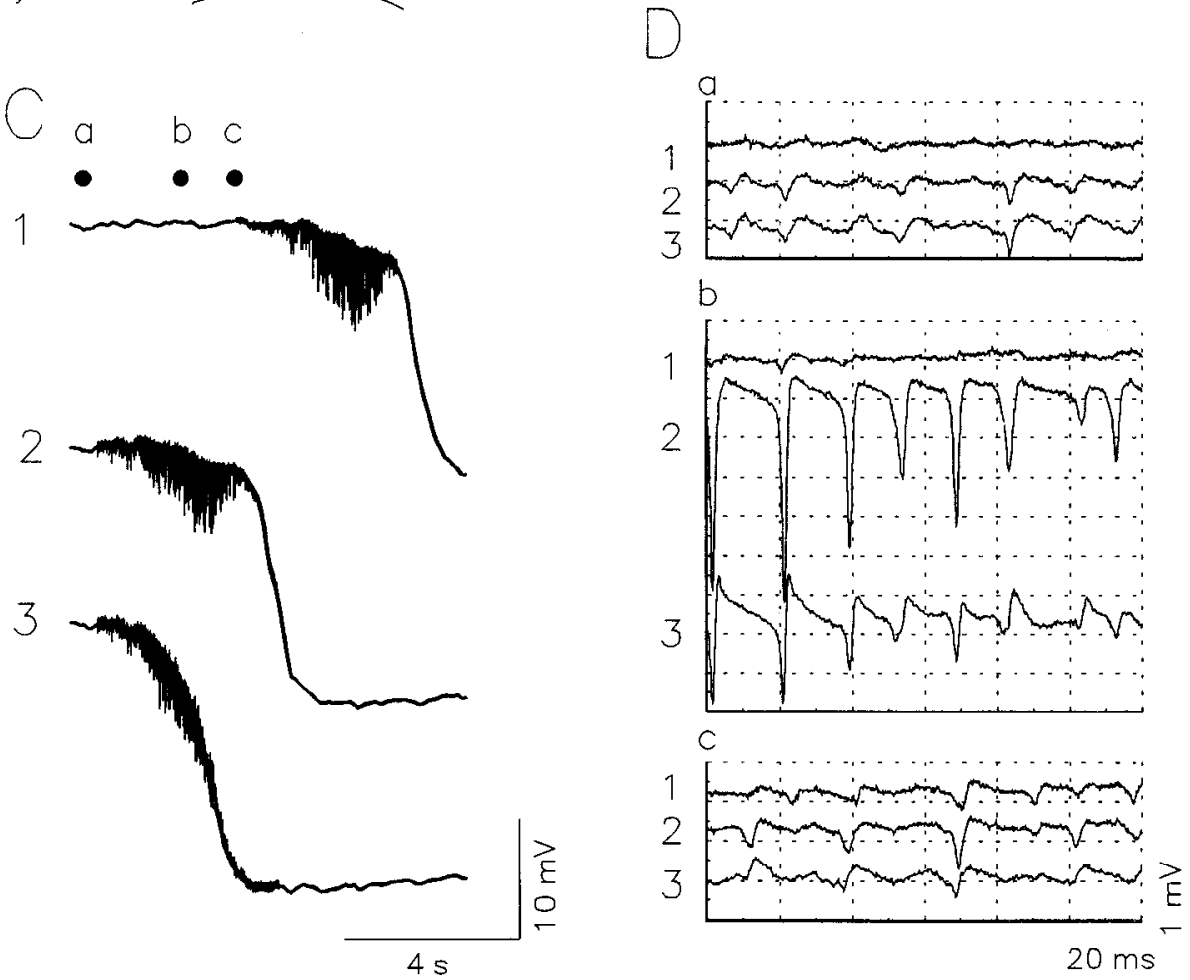

different depths along one track. As previously reported (Herreras and Somjen, 1993a,b), the $\Delta V_{o}$ shifts started in most preparations in st. radiatum $2-4 \mathrm{sec}$ earlier than in st. pyramidale (Fig. 3A). There was no such delay of the prodromal spike burst in st. pyramidale. In the experiment illustrated in Figure 3 the PS amplitudes grew in fact faster in st. pyramidale than in st. radiatum, but this was not replicated in other preparations.

There was a striking difference in the evolution of the waveform of the PSs between the two main layers. In st. pyramidale the PSs in a burst were always negative (Fig. $3 B$, Pos 1 , e 3 and Pos2, e2). In the proximal sublayer of st. radiatum what began as simple negative spikes turned into biphasic positive-negative signals and finally monophasic positive spikes as the $\Delta V_{0}$ developed (Fig. 3B, Pos2, e 3 and Pos3, e2). In the deeper layers, among the distal dendritic arbors, the PSs were predominantly positive all along (Pos3, e3). The changeover from negative to positive spikes in proximal st. radiatum coincided with the fast negative excursion of the DC potential at the onset of the SD wave in this stratum (seen only as a transient negative deflection in the AC-coupled trace) and it could not have been an artefact due to electrode slippage, because no sign of corresponding movement could be detected in the trace from electrode in st. oriens and/or st. pyramidale (Fig. 3, positions 2 and 3).

Positive spikes in st. radiatum could reach maximally $5 \mathrm{mV}$ in amplitude. They tended to still grow while the negative spikes in st. pyramidale were declining so that at certain times the positive spikes exceeded in amplitude the corresponding negative ones recorded more dorsally.

In a small number of preparations the $\Delta V_{o}$ in st. pyramidale preceded that in st. radiatum (Herreras and Somjen, 1993a,b). In these cases the prodromal spike bursts evolved differently, in that no positive spikes were seen in any of the layers. The bursts extinguished right before the $V_{o}$ shifted in the now leading st. pyramidale. Thus, in the absence of the negative $\Delta V_{o}$ the PSs in st. radiatum do not change from negative to positive.

\section{Current source-density (CSD) analysis and phase relations of PSs}

Figure 4 illustrates currents calculated from the potential records shown in Figure 3. The st. oriens (Pos1) appears to provide current sources for the current sinks in st. pyramidale. By contrast, in the proximal sublayer of st. radiatum (Pos 3) the initial sinks give way to sources. These sources grow in amplitude while the sinks in st. pyramidale decline in amplitude. The transition from sink into source is illustrated in greater detail in Figure $4 B$. Farther ventrally, among the dendritic arbor and neuropil of distal st. radiatum, the spikes were always of positive polarity (Fig. $4 B, \phi_{\mathrm{e} 3}$ ).

In Figure $5 A$, the field potentials $\left(\phi_{\mathrm{e} 1}-\phi_{\mathrm{e} 3}\right)$ and the calculated currents for e2 (lower row, st. pyramidale) are shown for the 

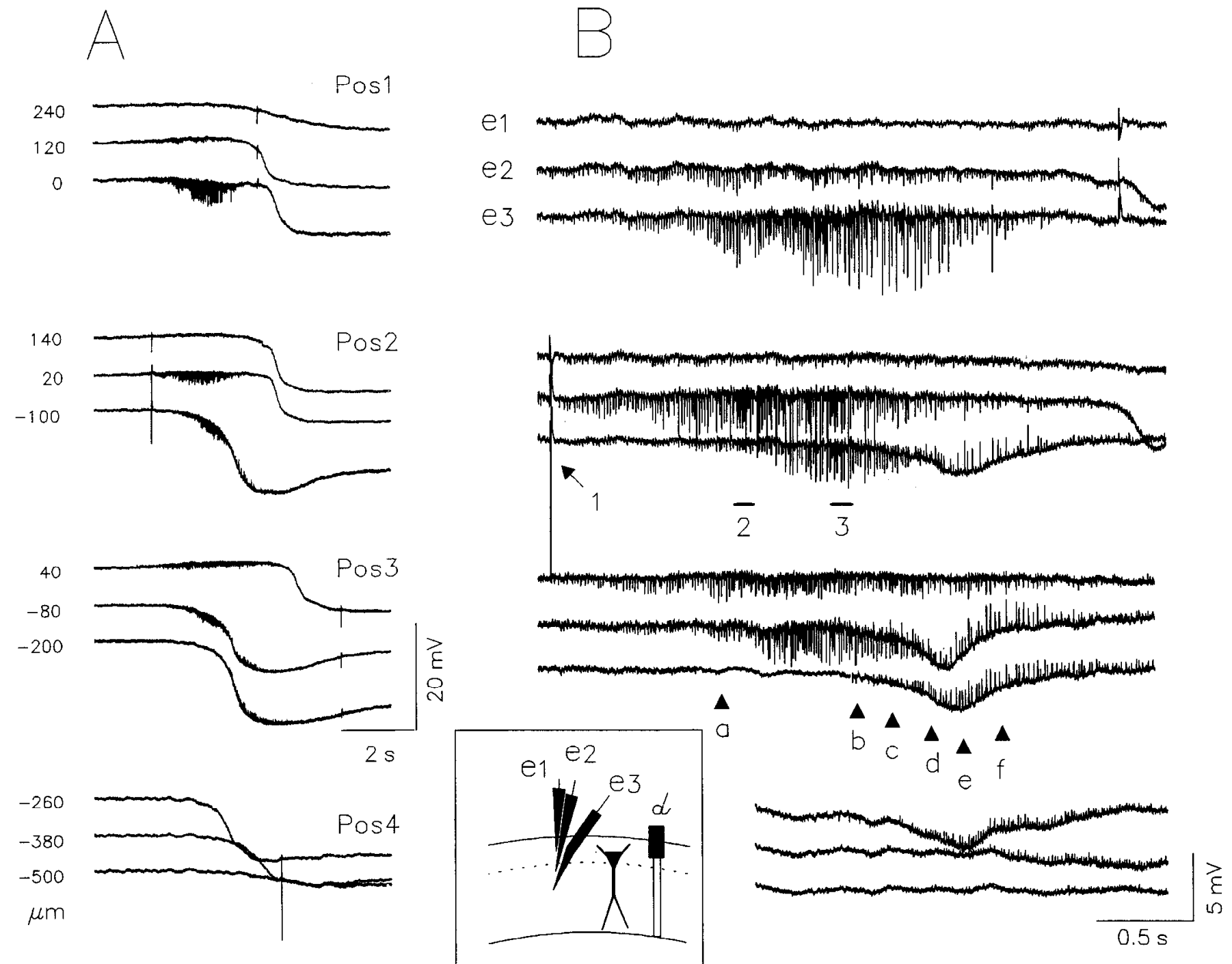

Figure 3. Pattern of PS bursts in different cytoarchitectonic layers. DC-coupled $(A)$ and AC-coupled $(B)$ recordings were made with an assembly of three electrodes fastened as shown by the inset. The triple assembly was repeatedly advanced in the tissue. Numbers labeling the traces under $A$ refer to depth of the recording site: zero depth corresponds to the st. pyramidale, where the evoked negative PS was of maximal amplitude; positive numbers refer to more superficial, and negative numbers to deeper locations. Recordings are lined up taking as a reference the fast DC drop. Note in $A$ the delayed occurrence of the DC drop in st. pyramidale and oriens as compared to the st. radiatum. Observe pure negative PSs in st. pyramidale $(P o s I, e 3)$ and negative to positive transition in proximal st. radiatum $(P o s 3, e 2)$ during the DC drop (reflected as an ample negative bump). Middle to lower st. radiatum only developed positive PSs $(P o s 3, e 3)$. Large positive PSs are still present at a time when negative PSs are almost subsided at upper positions. Letters $a-f$ underneath third set of traces in $B$ refer to sample spikes and CSD traces of Figure $4 B$, and numbers $1-3$ mark fragments cxpanded in Figure 5.

selected segments labeled 1-3 in Figure $3 B$ (position 2). Column 1 of Figure $5 A$ illustrates normal orthodromic evoked potentials recorded simultaneously by the three electrodes, and the CSD calculated for the middle electrode. Negative spikes always occur earlier in the proximal st. radiatum than in st. pyramidale (Fig. 5B, evoked traces). The orthodromic spike sink was found up to $200 \mu \mathrm{m}$ below cell somata. Earlier detailed CSD analysis of normal orthodromic evoked responses in CA1 (Herreras, 1990) revealed that a synaptically triggered sink in st. radiatum, identified as a dendritic action potential, appears to be an obligatory antecedent of the orthodromic soma-axonal impulse. This obligatory sequence was not evident in the recordings of spontaneous PSs in the prodromal spike bursts. Usually the peaks of the negative PSs recorded in st. pyramidale and in proximal st. radiatum coincided (Fig. 5B, spontaneous), but slight shifts, with either the one or the other layer leading, did occur. The lead could change even within the same burst. In the segments 2 and 3 (Fig. $5 A$ ) the PS sinks in st. pyramidale were of a comparable magnitude whether or not large negative PSs appeared at the st. radiatum. At the time chosen in scgment 3, that is, before $V_{o}$ begins to fall in st. radiatum, also spike sinks are calculated in this stratum (compare first half of Pos2 and Pos3 in Fig. 4A) and therefore a simultaneous spike sink is generated in both strata.

\section{Interaction between evoked responses and prodromal spike bursts}

Ortho-and antidromic responses were evoked before and during spike bursts. Single orthodromic stimuli had little effect on spike bursts, but double stimuli strongly inhibited them. In Figure $6 \mathrm{~A}$ 

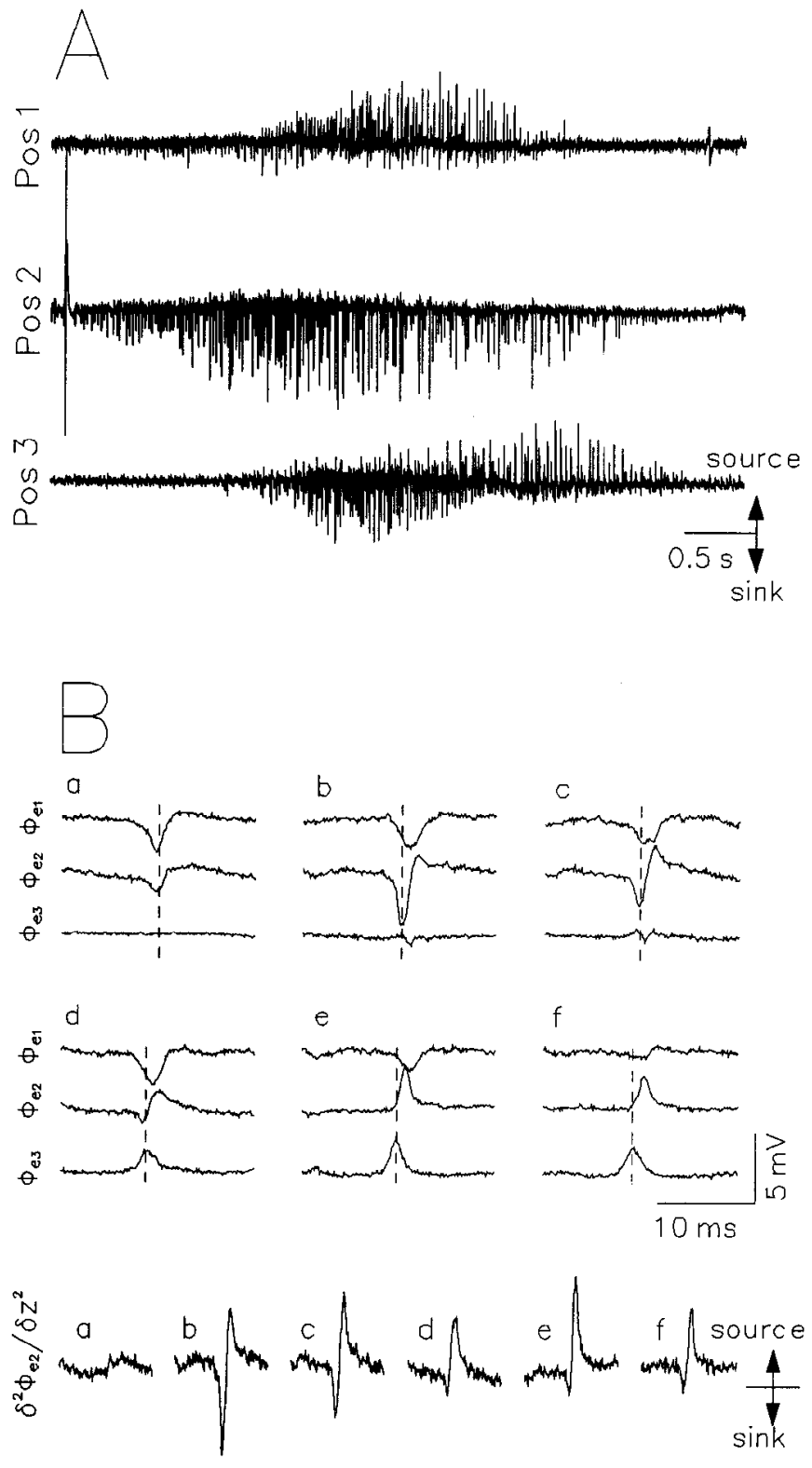

Figure 4. Localization of transmembrane currents during the PS burst by current source-density (CSD) analysis. $A$, CSD calculated for the center trace of triple recordings shown in Figure $3 B$. Position 1 , st. oriens; 2 , st. pyramidale; 3 , proximal st. radiatum. Large spike sources develop in the st. radiatum as DC plunges negative in this layer. $B$, Expansions of the sample spikes labelled in Pos 3 of Figure $3 B$, and recorded in st. pyramidale $\left(\phi_{e l}\right)$, and proximal and mid st. radiatum $\left(\phi_{e 2}\right.$ and $\phi_{e s}$ ), and the calculated current (bottom row) for the intermediate electrode. Note the progressive unmasking of the source component as the sink decreases. Broken lines are drawn to facilitate comparisons of timing.

a double shock delivered during the burst depressed the PS amplitudes for considerable period. In Figure $6 B 2$ continually repeated twin stimuli altogether prevented the spike burst. By varying the intensity and the frequency of the orthodromic stimulation it was shown that the degree of depression varies with the intensity of the input. Stimuli subthreshold for the PS and frequencies below $1 \mathrm{~Hz}$ were ineffective. The timing of orthodromic stimuli commencement was important as well, the more pulses delivered before the prodromal PS burst the more severe its decline. An example is illustrated in Figure $6 C$, where single
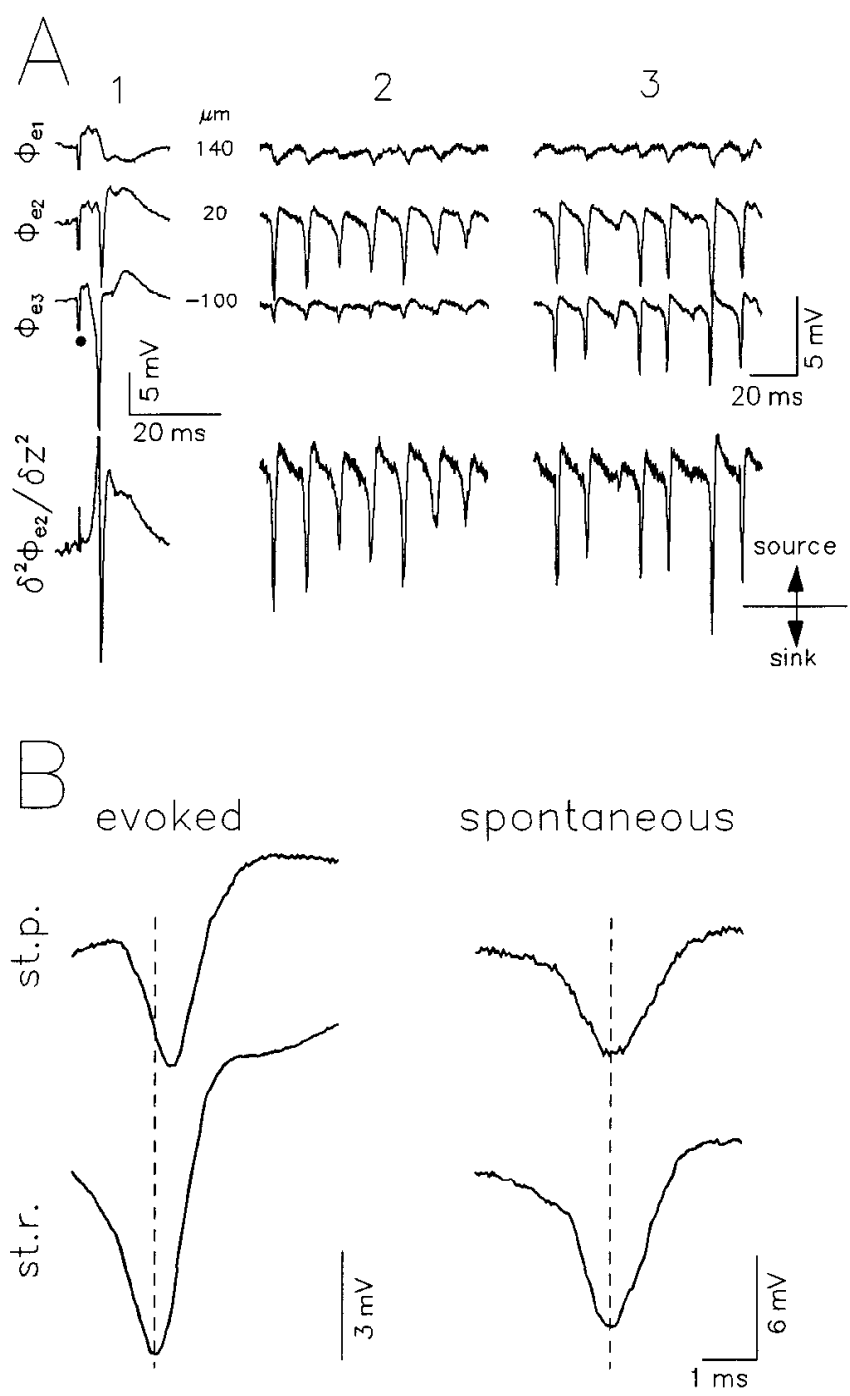

Figure 5. Comparing time relations of orthodromically evoked PS, and spontaneous spikes within prodromal spike bursts. $A$, Fragments of the selected field potentials $\left(\phi_{e}-\phi_{e 3}\right)$, in Figure $3 B$ and the calculated currents (lower row) in st. pyramidale. $I$ shows the typical orthodromic potentials evoked in the three layers and the somatic current. In 2 and 3 , only pure sinks of equivalent magnitude were calculated in the cell body layer regardless of the activity in the adjacent strata. In this and the next figures, solid circles mark the artifact (truncated) during evoked potentials. $B$, Comparison of the PS peak latencies in st. pyramidale and radiatum ( $90 \mu \mathrm{m}$ below) during orthodromic activation (evoked) and in a sample PS within the prodromal burst (spontaneous). Compare the characteristic headway of dendritic PS in st. radiatum during evoked PSs versus the simultaneous occurrence in both layers in the later case.

orthodromic pulses delivered at $2 \mathrm{~Hz}$ were started only $2 \mathrm{sec}$ before the burst outset, and no clear decline of PSs was noticed. Therefore, some cumulative event resulting by repetitive orthodromic activation counteracts the necessary synchronization for the spontaneous PS buildup.

Not only did orthodromic activation modify the SD-related spontaneous activity, the oncoming SD wave influenced orthodromic transmission well before the arrival of the $\Delta V_{o}$ shifts. Both depression and hypertransmission were observed in various phases. In the example of Figure $6 C$ the orthodromic PS responses are depressed before the onset of the $\Delta V_{o}(40-70 \%$ reduction within 1-3 sec before PS burst arrived, $n=10$ ). In Figure $6 C$ it is, however, also apparent that during the burst the 

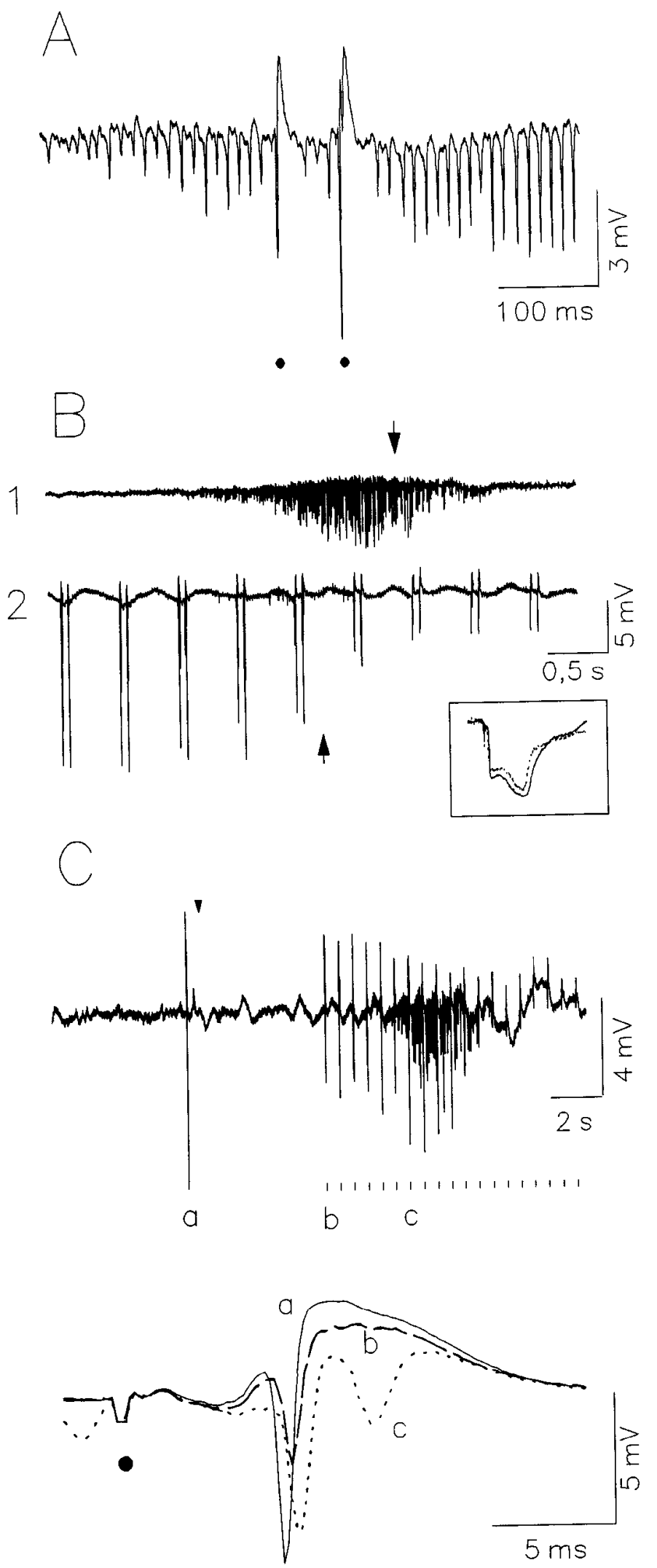

Figure 6. Interaction of orthodromic evoked responses and prodromal spike bursts. All recordings are AC-coupled recordings from st. pyramidale. $A$, Two orthodromic stimuli marked by dots, delivered during a spike burst preceding a $\Delta V_{o}$ shift strongly inhibited spontaneous spikes. $B$, Initial events of two successive SD episodes in the same preparation. I, Without orthodromic stimulation. 2, During continuously repeated orthodromic evoked PS that is initially profoundly depressed (dashed trace) increases again and develops double spikes (dotted trace), suggesting hyperexcitability. It may be objected that this could have been the result of the facilitation known to occur normally during repetitive stimulation. That the approaching SD adds an influence not normally present, is shown in Figure 7. Figure $7 A$ illustrates responses to single stimuli at rest (left column) and before the arrival of a $\Delta V_{o}$ shift (right column) recorded near (traces 2 and 3) and far (traces 1) from the SD wavefront (see electrode arrangement in Fig. $2 A$ ). Multiple orthodromic PSs appear only in the region already under the influence of the approaching SD wave (right column, traces 2 and 3). Figure $7 B$, from another experiment, illustrates the difference of the effect of repetitive orthodromic volleys at rest and in the prodromal stage of SD. Surprisingly, the latency of the orthodromic PSs increased even though multiple spike firing was enhanced, suggesting increased excitability.

Antidromic stimuli did not influence the prodromal spike bursts. The antidromic spikes became gradually suppressed either during or before the onset of the spike burst (Fig. $8 B$ ). Sometimes (two out of seven experiments), however, there was a moderate augmentation of the antidromic spikes before they were extinguished (Fig. 8A). The antidromic spikes were not blocked while being conducted in the alveus, because they were still normal at the moment when a $\Delta V_{o}$ shift invaded the region of the stimulating electrode. Earlier observations (Herreras and Somjen, 1993b) have shown that in alveus and st. oriens the increase of $\left[\mathrm{K}^{+}\right]_{o}$ during SD is slight and probably not sufficient to disturb impulse conduction.

\section{The roles of $\left[\mathrm{K}^{+}\right]_{\circ}$ and of synaptic transmitters in $S D$}

The glutamate receptor blocking drugs, DNQX, or CPP, or both, were administered through the dialysis fiber prior to the perfusion of high- $\mathrm{K}^{+}$, creating an aura of tissue around the dialysis probe that was affected by the drugs. The reference barrel of $\mathrm{K}^{+}$-sensitive electrodes was used to record electric potential. As expected, $100 \mu \mathrm{M}$ DNQX but not CPP abolished orthodromic field potentials (Herreras et al., 1989). As also rcported earlier (Herreras and Somjen, 1993b), DNQX by itself did not alter the SD waves whereas CPP decreased the frequency of their appearance and abolished the somatic DC wave and the late portion of the dendritic SD wave (phase II; see Herreras and Somjen, 1993b) without preventing their appearance or propagation. The combined administration of CPP and DNQX (Fig. $9 \mathrm{~A}$ ) yielded the same result as CPP alone (three and two animals, respectively).

Examination of the enlarged early parts of the recordings show that the combined administration of DNQX and CPP did not

twin orthodromic stimuli. A complete blockade of the prodromal PS burst ensued orthodromic activation (twin pulses, $2 \mathrm{~Hz}$ ). Large vertical strokes are the orthodromic PSs (stimulus artifacts have been deleted to uncover PS). The arrows mark, as a reference, the onset of the $\Delta V_{o}$ shift in the st. radiatum. The inset shows superimposed low-gain DCcoupled traces of the same two events, illustrating similarity of the $\Delta V_{o}$ shifts $(90 \mathrm{sec}$ and $28 \mathrm{mV}) . C$, Amplitude modulation of orthodromic responses during a prodromal spike burst. a marks a control orthodromic PS; at the arrowhead an SD episode was initiated by microejection of $\mathrm{K}^{+}$in a more caudal pipette and then orthodromic single pulses (marked by the dashes below) reinitiated $2 \mathrm{sec}$ before the prodromal PS burst arrived. In the lower part, expanded potentials of the responses labeled $a-c$. 

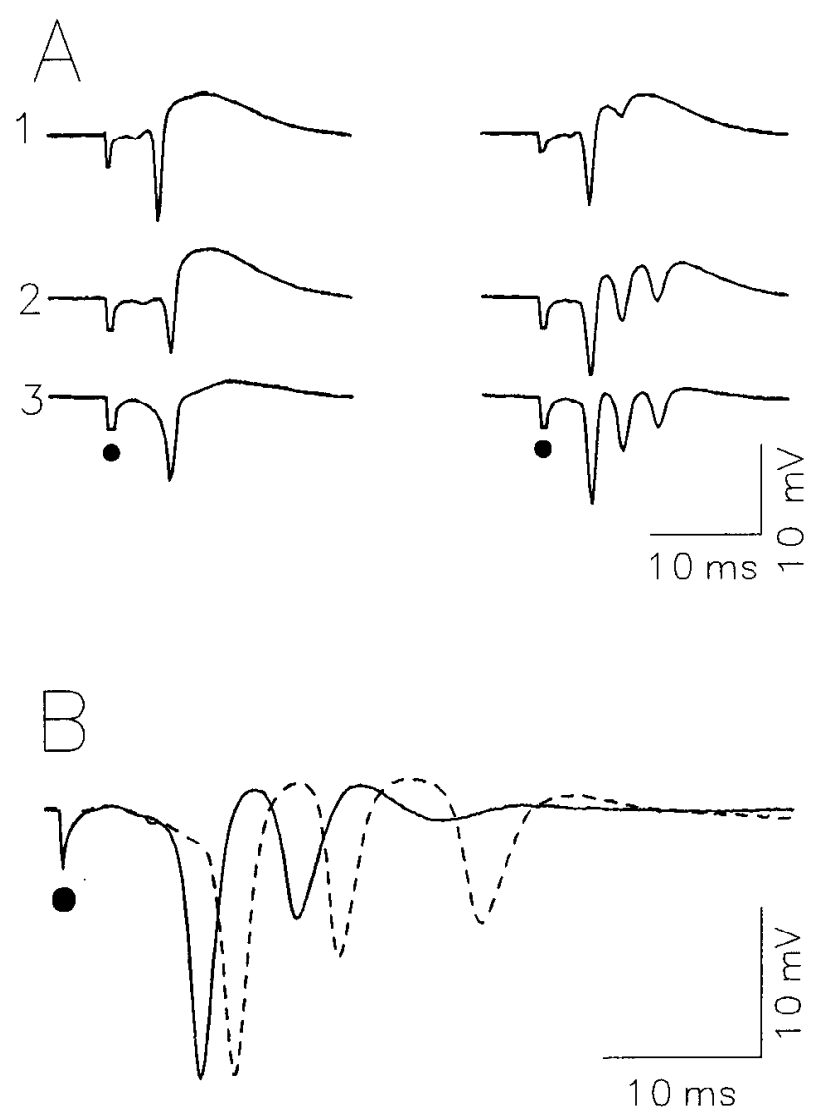

Figure 7. Hypertransmission during prodromal spike burst. $A$, Orthodromic responses recorded with three electrodes placed as shown in inset of Figure 2: 1 and 2 in st. pyramidale at $1 \mathrm{~mm}$ distance, 3 in proximal st. radiatum (region of dendritic action potential generation). Left column of traces, in SD-free control period; right column, when a prodromal spike burst has reached positions 2 and 3 but not yet position I. $B$, Superimposed orthodromic responses during a $2 \mathrm{~Hz}$ train recorded six (solid line) and $2 \mathrm{sec}$ before the arrival of a $\Delta V_{0}$ shift (broken line). The former was obtained once evoked potential variations stabilized within the stimulus train and the later at the usual time of prodromal burst. Note that a third PS still developed at the SD immediacy, but all of them are delayed as compared to instants earlier. Responses were recorded in the proximal st. radiatum, $90 \mu \mathrm{m}$ below the center of st. pyramidale.

block the prodromal spike bursts (Fig. 9B). Moreover, the spike burst began before $\left[\mathrm{K}^{+}\right]_{0}$ started to increase. These time relations were found consistently in $12 \mathrm{SD}$ episodes in three rats, as well as one trial with DNQX alone and two with CPP alone.

Since glutamate is not the only excitatory transmitter substance in hippocampus, we also tested the effect of blocking all synapses. $\mathrm{CoCl}_{2}$ (10 mM), $\mathrm{CdCl}_{2}$ and $\mathrm{NiCl}_{2}$ (1 mM each) were added to the dialysis fluid. When orthodromic evoked potentials recordcd $400 \mu \mathrm{m}$ from the dialysis probe were suppressed, SD was provoked by high-K $\mathrm{K}^{+}$solution ejected from another micropipette farther rostral. SD waves with the typical $\Delta V_{o}$ shifts invaded the area where synaptic transmission was blocked and they were preceded by prodromal spike showers as usually (one animal).

We also tested the effect of the $\mathrm{Na}^{+}$channel blocker TTX (10 $\mu \mathrm{M}$ ) in three animals. Confirming earlier reports (Sugaya et al., 1975; Tobiasz and Nicholson, 1982; Herreras and Somjen, 1993b), SD waves could be provoked within the region infiltrated with TTX and SD waves propagated normally out of the

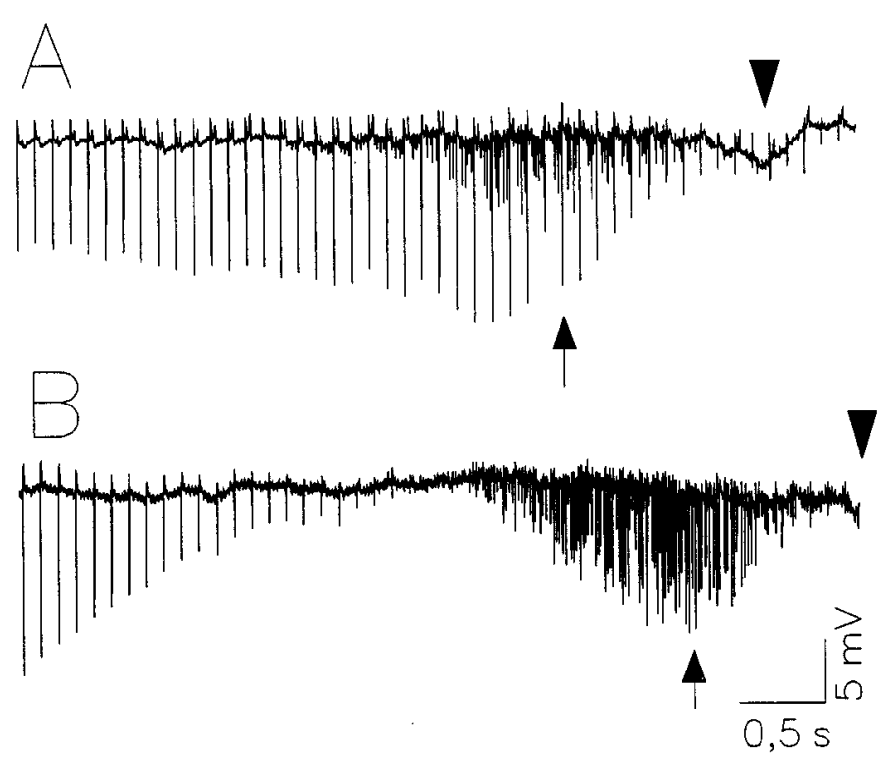

Figure 8. Modulation of antidromic spike amplitude by prodromal phase of SD in two different animals $(A$ and $B)$. Both AC-coupled recordings in st. pyramidale, stimulation of alveus at $10 \mathrm{~Hz}$. Arrows mark the onset of the $\Delta V_{o}$ shifts at the st. radiatum and arrowheads at the st. pyramidale. Large vertical strokes in $A$ and initial ones in $B$ correspond to antidromic PSs.

affected region. Prodromal spike bursts and sawtooth wavelets were, however, absent within the region influenced by TTX but reappeared when the SD wave emerged from the TTX block.

\section{Discussion}

The electrical activity recorded before the onset of the negative $\Delta V_{o}$ shift was highly reproducible. Even though SD waves can occur when the prodromal spike burst is suppressed by TTX, the otherwise completely predictable appearance of the burst indicates that something in the functional properties of neurons is changing well before the onset of the $\Delta V_{o}$ shift. These early changes must be considered as integral components in the evolution of the SD process.

Detailed examination of the early events that precede the $\Delta V_{o}$ shift revealed several striking features. One is the regular frequency shared by both the sawtooth-like wavelets and the PSs in a spike burst and synchronized over considerable distances within the tissue. Second, that the spike bursts arc indcpendent of synaptic transmission. Third, the $\Delta V_{o}$ shifts can occur without the antecedent spike burst. Fourth, that synaptic transmission and antidromic conduction are altered well before the onset of the profound depolarization signaled by the $\Delta V_{o}$ shift. Fifth, that the electrical signs of the oncoming $\mathrm{SD}$ wave begin before $\left[\mathrm{K}^{+}\right]_{0}$ begins to rise.

\section{The sawtooth wavelets are the pacemaker of the spike burst}

The following characteristic observations suggest that the sawtooth wavelets are pacing the PSs in the bursts. The spikes gradually grow out of the sawtooth wavelets. Spikes can be seen to take off from sawtooth wavelets as a sudden steepening of the voltage deflection (Figs. $1 B 2,2 B$ ). In any given experiment the frequency of the sawtooth wavelets was the same as that of spikes in a burst. And, whenever a PS in a burst failed, it did so at all recording electrodes, and a sawtooth wavelet could be discovered underneath the missing spike (Fig. 1B2). The next 
spike then followed at the double of the usual interval, that is, without resetting the rhythm. The biophysical mechanism responsible for the pacemaker activity is discussed below.

\section{Spontaneous PSs indicate electrically mediated synchronization of neuron impulses}

PSs of several millivolts amplitude can arise only by the exactly in-phase firing of a large number of neurons. The synchronization of the firing in the prodromal spike bursts was not mediated by synaptic mechanisms because the bursts appeared normally even when synaptic transmission was blocked. This leaves electric interaction as the mechanism responsible for simultaneous firing. Electric interaction among cells could be achieved by current flowing between cells, that is, ephaptically, or through electrically patent intercellular junctions, most probably gap junctions. The spontaneous PSs that are fired during tonic seizures are probably similarly electrically synchronized. This type of activity has earlier been termed "lockstep firing" (Somjen et al., 1985).

High-frequency electrical activity, synaptic noise, and impulse firing ahead of SD-related $\Delta V_{o}$ shifts have been recorded from individual cells in the neocortex and hippocampus (Morlock et al., 1964; Rosenblueth and Garcia-Ramos, 1966; Ichijo and Ochs, 1970; Muñoz, 1970; Higashida et al., 1974; Somjen and Aitken, 1984; Haglund and Schwartzkroin, 1990). The large PSs resulting from lockstep firing are, however, peculiar to hippocampus and dentate gyrus. The tight packing of cells in st. pyramidale of the cornu ammonis is believed to favor ephaptic interaction among cells and this could favor hypersynchrony of action potentials (Taylor and Dudek, 1982a; Snow and Dudek, 1984). However, it does not solve the initial necessary firing. The prompt reactivation of previously suppressed fast activity when a wave of SD emerges from a region infiltrated by TTX shows that distant PSs are not necessary to initiate fast activities.

Earlier CSD studies (Herreras, 1990) have shown that orthodromically transmitted PSs arise first in the proximal zone of apical dendritic shafts, before the PS in the layer of cell somata. By contrast, the PSs in prodromal bursts usually appeared in phase along the entire expanse of somata and proximal dendrites (Fig. $5 B$ ). This suggests widely spread depolarization reaching the threshold simultaneously. This notion is also reinforced by the similarly in-phase appearance of the sawtooth wavelets. We propose that the sawtooth activity is the extracellular representation of a synchronized oscillatory membrane process. Spike bursts may be initiated when some additional excitatory influence arises as the SD process evolves and enables the initially subthreshold oscillations to trigger impulses.

\section{Synchronization over long distances probably requires intercellular electrical communication}

If SD propagatcs in hippocampus with velocity of $80-125 \mu \mathrm{m} /$ sec (Herreras and Somjen, 1993a) and the earliest prodromal fast activity begins 5-8 sec ahead of an advancing $\Delta V_{0}$ shift, then neurons over a distance of $0.4-1.0 \mathrm{~mm}$ must be simultaneously engaged in the fast activity. It seems unlikely that primary synchronization over such distances is achieved by ephaptic interaction, that is to say, current flowing in and out of the membranes of adjacent cells, especially unlikely with view of the small but rather constant average amplitude of the sawtooth wavelets that continue for seconds before the eruption of the spike shower (Figs. 1B, 2).
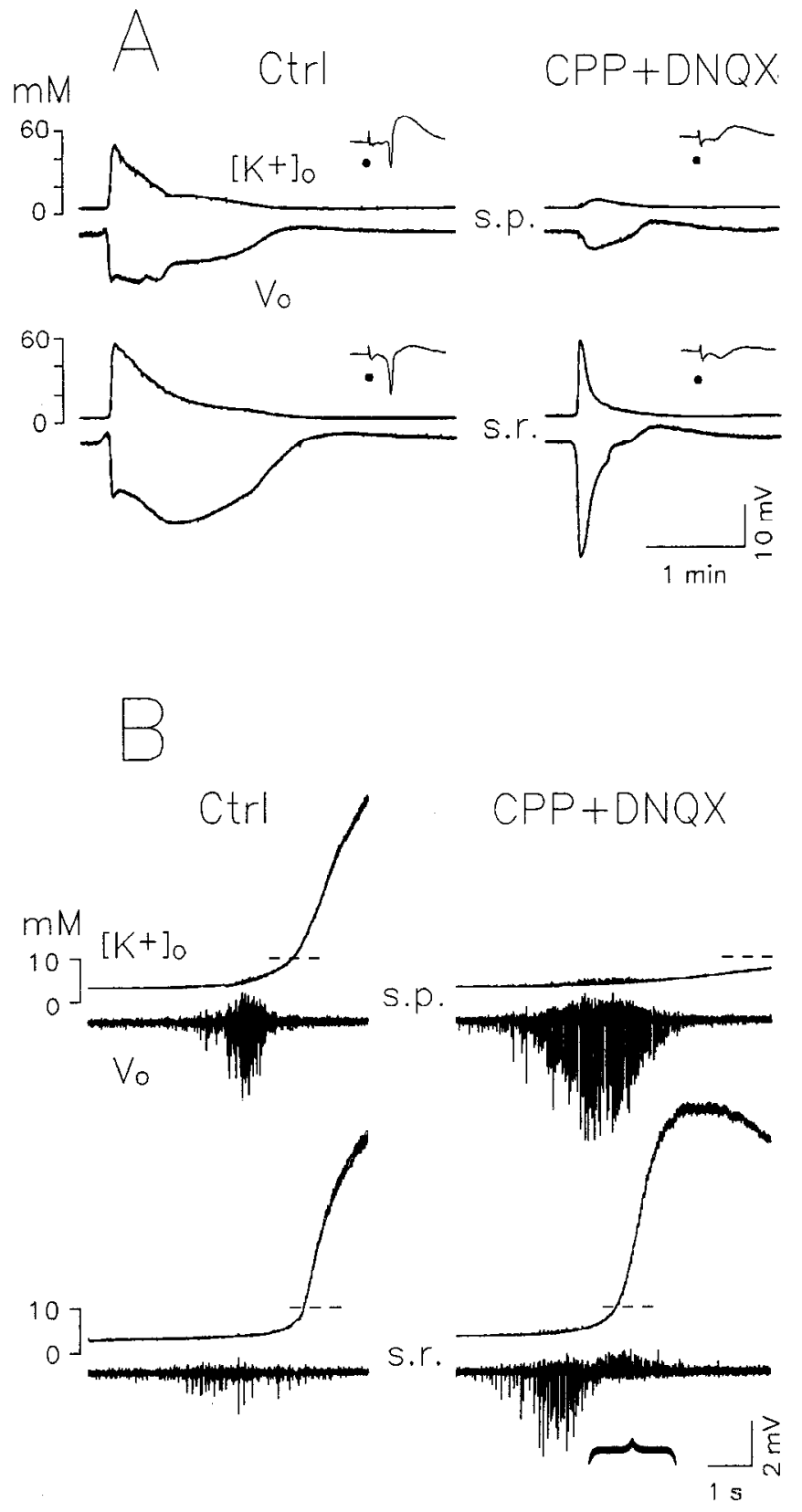

Figure 9. The effect of blockade of glutamate receptors on SD, and the timing of the increase of extracellular potassium $\left(\left[\mathrm{K}^{+}\right]_{o}\right)$. Recording with double-barreled ion-selective electrode of $\left[\mathrm{K}^{+}\right]_{o}$ and potential $\left(V_{o}\right)$. $A$, DC-coupled traces from st. pyramidale (above) and st. radiatum (below). Left column, SD episodes provoked during initial control period; right column, during the action of CPP and DNQX. Insets show sample orthodromic evoked potentials, confirming blockade of synaptic transmission. $B$, Expanded records of initial phase of the SD episodes shown in $A$. The voltage traces are AC coupled. Prodromal PS burst initiates before $\left[\mathrm{K}^{+}\right]_{0}$ rises. Dashed horizontal lines indicate $10 \mathrm{mM}\left[\mathrm{K}^{+}\right]_{0}$ level. The bracket points to the phase of positive PSs in st. radiatum. Note linear scale of $\left[\mathrm{K}^{+}\right]_{o}$, transformed by computer from recorded voltages.

Much more probably, the early synchronization is brought about through electrical current by way of gap junctions. In the resting state CA1 pyramidal neurons may be only weakly connected (MacVicar and Dudek, 1981; Taylor and Dudek, 1982b). We have already suggested that among the earliest stages of the evolution of SD is the opening of normally closed electrical 
junctions among cells (Somjen et al., 1992). Many agents known to change during SD can also alter gap junctions conductance (see Bennett et al., 1988, for a review). A simple way to increase electrical junctions conductance may occur during the transient alkaline shift known to occur at the initial stages of SD (Mutch and Hansen, 1984). In fact, alkaline treatments have been shown to increase both neuronal dye coupling and the ability of CAl pyramidal neurons to generate burst firing (Church and Baimbridge, 1991).

A most peculiar and remarkable observation in the present study is the very high and fixed frequency of the fast activities. Such an across-animal constancy indicates that it results from an intrinsic property of either individual cells or a cell aggregate. Interestingly, synchronous and rhythmic firing has been considered an emergent property of electrotonically coupled cells due to regenerative feedback (Getting and Willows, 1974; see also Sherman and Rinzel, 1992). We consider the possibility that, as it has been shown for other cellular systems (see, e.g., Christie et al., 1989), the low-pass filtering capability of electrical synapses of an appropriate patterned firing in one cell may generate an oscillatory membrane potential in the coupled cells, which in the end may result in synchronous firing.

Positive extracellular spikes and large current sources may also be generated by current flowing through intercellular junctions

Positive extracellular spikes are usually interpreted as representing passive sources for current flowing into active sinks on the membrane of the same extended cellular process. In the case of recordings such as those shown in Figures 3 and 4 this explanation leads to difficulty, because the sources of the proximal dendritic region grow in amplitude at a time when the sinks in the cell body layer are decreasing.

The paradox of a source greatly exceeding in amplitude a corresponding sink could be explained in one of three ways. Either (1) the source is "active" and the sink is "passive," or (2) the source is much more limited in extension than the sink (i.e., the current is more dense in the source region), or (3) the source supplies current to a sink located elsewhere and not seen by the recording array.

For the source to be "active" we would have to have a sizeable outward membrane current. The sources in st. radiatum developed while this region showed the large negative $\Delta V_{o}$ signaling massive depolarization of, at least, the dendritic domain. In this stage ion gradients between cytosol and interstitial fluid decrease or approach zero, but they do not reverse (Kraig and Nicholson, 1978; Czéh et al., 1993). In the absence of a driving force, there cannot be an outward membrane current.

It is also unlikely that the source region would be less extensive than the sink region of the same cells. When the positive spikes attain maximal amplitude the sources extend over much of the dendritic region while the dwindling sinks remain limited to the soma region.

By exclusion, the most likely explanation is that the sources were supplying current to an unseen sink located on membranes that are not part of the pyramidal cells generating the source. We suggest that the sinks are on neurons located ahead of the propagating $\Delta V_{o}$ shift, on neurons that are not yet depolarized but are already firing in the early phase of the spike burst. This hypothesis implies, once more, electrical continuity of the cytoplasm of adjacent cells.

\section{Neuronal excitability changes before extracellular signs of $S D$}

Some authors have reported a neuronal silence ahead of a moving SD (Morlock et al., 1964; Higashida et al., 1974), which in cortical cells may be as long as $11 \mathrm{sec}$. This early cessation of activity is paralleled in our experiments by the decreased orthodromic PS before the moving burst of PSs arrived to the electrode (Fig. 6). So far, we have no clues as to the origin of this modulation, but the changed responses to orthodromic and antidromic stimulation (Figs. 6-8) as well as the unexpected increased latency for the orthodromic PSs during the burst period do indicate, that the state of the neuron membranes is altered well before the onset of the profound depolarization signaled by the $\Delta V_{o}$ shift.

The mutual interaction of orthodromic volleys with prodromal spike bursts may be due to changing electrical properties of the neuron membranes. The beginning depolarization of pyramidal cell dendrites seems to slow synaptic transmission but at the same time to enhance multiple firing in response to single orthodromic volleys (Figs. $6 \mathrm{C}, 7 \mathrm{~B}$ ). On the other hand, repetitive synaptic input reduces membrane resistance, and this could wcaken clcctric coupling among cells (ref. Spira and Bennett, 1972), thus suppressing the prodromal spike bursts (Fig. 6B). This interpretation may be reinforced by the heavier prodromal bursts recorded after blockade of Glu receptors (Fig. 9B), which presumably reduced overall cell conductance by curtailing the background excitatory synaptic bombardment.

\section{Neither elevated $\left[\mathrm{K}^{+}\right]_{\mathrm{o}}$ nor the release of a transmitter agent is responsible for the initiation of $S D$}

The role of elevated $\left[\mathrm{K}^{+}\right]_{o}$ has been controversial for many years (Grafstein, 1956; Marshall, 1959; Haglund and Schwartzkroin, 1990). Recently Lehmenkühler (1990) and we (Herreras and Somjen, 1993b) have reported findings that are difficult to reconcile with the idea that the release of $\mathrm{K}^{+}$ions is the trigger for $\mathrm{SD}$ initiation. Records such as the ones illustrated in Figure $9 B$ confirm that the earliest phase of the SD process begins before $\left[\mathrm{K}^{+}\right]_{0}$ starts to rise. This figure also shows that, while the blockade of glutamate receptors partially suppressed the $\Delta V_{o}$ and $\Delta\left[\mathrm{K}^{+}\right]_{0}$ in st. pyramidale, the prodromal spike burst was enhanced instead of inhibited. This, of course does not negate the fact that the eventual rise of $\left[\mathrm{K}^{+}\right]_{o}$ to levels not equalled in any other pathological condition is an integral part of the evolution of SD and must by necessity powerfully feed back on the functioning of the cells that have released the $\mathrm{K}^{+}$ions.

A similar conclusion emerges with regard to the roles of synaptic transmitter substances. There is no doubt that during SD glutamate and other transmitters are released from cells (Szerb, 1991; Fabricius et al., 1993). Much has been made of the fact that drugs that block NMDA receptors retard the onset and propagation of SD (Gorelova et al., 1987; Hansen et al., 1988; Nellgärd and Wieloch, 1992). We have confirmed the strong modulation of the timing and the $\Delta V_{o}$ waveform by such agents (Fig. 9; see also Herreras and Somjen, 1993b). The fact remains, however, that these blocking drugs did not prevent the appearance of SD waves. Blockade of calcium currents by divalent cations was similarly unsuccessful in preventing SD (see also Jing et al., 1993). We conclude that transmitters released from storage modify the evolution of the SD process, but they are not responsible for its initiation. 


\section{Final remarks}

Even though the discharge of impulses is not required for the initiation or the propagation of SD, the impulse shower does regularly appear at its beginning. The mechanism that gives rise to the impulse discharge may well have a key role in the evolution of SD. The widely spread synchronization seems best explained by electrical continuity that could be provided by gap junctions. Effective communication by way of quasi-syncytial nets has been demonstrated in other systems, for example in the spread of so-called calcium waves in cell cultures (CornellBell et al., 1990; Dani et al., 1992; Finkbeiner, 1992). Patent gap junctions may provide a path not only for electric current and for ions but also for intracellular "second" messengers and other active ingredients in cytosol. SD has frequently been interpreted as a diffusion-reaction process whose velocity of spread is governed by the rate of the reaction, which could involve the release of some substance from cells that then acted on the cell membrane of adjacent cells. As there are reasons for doubting a decisive role of either $\mathrm{K}$ or of glutamate, we are proposing an alternative hypothesis, involving the exchange of chemical signals not through the interstitial space but by way of gap junctions. The autocatalytic reaction so initiated would alter the membrane from the inside, instead of acting on receptors on the outside. This proposal is admittedly based on indirect evidence, and we offer it mainly as a catalyst of further inquiry.

\section{References}

Bennett MVL, Verselis V, White RL, Spray DC (1988) Gap junctional conductance: gating. In: Gap junctions (Hertzberg EL, Johnson RG, eds), pp 287-304. New York: Liss.

Brinley FJ, Kandel ER, Marshall WH (1960) Potassium outflux from rabbit cortex during spreading depression. J Neurophysiol 23:246256.

Bureš J, Křivánek J (1960) Ionic movements in the brain as studied with the aid of washing cortical surface with an epidural cannula. Physiol Bohemoslov 9:488-493.

Bureš J, Burešová O, Křivánek J (1974) The mechanism and application of Leão's spreading depression of electroencephalographic activity. Prague: Academia.

Christie MJ, Williams JT, North RA (1989) Electrical coupling synchronizcs subthreshold activity in locus coeruleus in vitro from neonatal rats. J Neurosci 9:3584-3589.

Church J, Baimbridge KG (1991) Exposure to high-pH medium increases the incidence and extent of dye coupling between rat hippocampal CA1 pyramidal neurons in vitro. J Neurosci 11:3289-3295.

Cornell-Bell AH, Finkbeiner SM, Cooper MS, Smith SJ (1990) Glutamate induces calcium waves in cultured astrocytes: long-range glial signaling. Science 247:470-473.

Czéh G, Aitken PG, Somjen GG (1993) Membrane currents in CA1 pyramidal cells during spreading depression (SD) and SD-like hypoxic depolarization. Brain Res 632:195-208.

Dani JW, Chernjavsky A, Smith SJ (1992) Neuronal activity triggers calcium waves in hippocampal astrocyte networks. Neuron 8:429440.

Fabricius M, Jensen LH, Lauritzen M (1993) Microdialysis of interstitial amino acids during spreading depression and anoxic depolarization in rat neocortex. Brain Res 612:61-69.

Finkbeiner S (1992) Calcium waves in astrocytes-filling in the gaps. Neuron 8:1101-1108.

Freeman JA, Nicholson C (1975) Experimental optimization of current source-density technique for anuram cerebellum. J Neurophysiol 38:369-382.

Getting PA, Willows OD (1974) Modification of neuron properties by electrotonic synapses. II. Burst formation by electrotonic synapses. J Neurophysiol 37:858-868.

Gorelová NA, Korelová VI, Amemori T, Pavlik V, Bureš J (1987)
Ketamine blockade of cortical spreading depression in rats. Electroencephalogr Clin Neurophysiol 66:440-447.

Grafstein B (1956) Mechanism of spreading cortical depression. J Neurophysiol 19:154-171.

Haglund MM, Schwartzkroin PA (1990) Role of Na-K pump potassium regulation and IPSPs in seizures and spreading depression in immature rabbit hippocampal slices. J Neurophysiol 63:225-239.

Hansen AJ, Lauritzen M, Wielloch T (1988) NMDA antagonists inhibit cortical spreading depression but not anoxic depolarization. In: Frontiers in excitatory amino acid research (Cavalheiro EA, Lehmann JL, Turski L, eds), pp 661-666. New York: Liss.

Herreras O (1990) Propagating dendritic action potential mediates synaptic transmission in CAl pyramidal cells in situ. J Neurophysiol 64:1429-1441.

Herreras O, Somjen GG (1993a) Propagation of spreading depression among dendrites and somata of the same cell population. Brain Res 610:276-282.

Herreras O, Somjen GG (1993b) Analysis of potentials shifts associated with recurrent spreading depression and prolonged unstable $\mathrm{SD}$ induced by microdialysis of elevated $\mathrm{K}^{+}$in hippocampus of anesthetized rats. Brain Res 610:283-294.

Herreras O, Somjen GG (1993c) Effects of prolonged elevation of potassium on hippocampus of anesthetized rats. Brain Res 617:194204.

Herreras O, Somjen GG (1993d) Prolonged unstable depression: a modified manifestation of spreading depression in rat hippocampus. In: Slow activity changes of the brain (Haschke W, Speckmann EJ, Roitbak AI, eds), pp 129-138. Boston: Birkhäuser.

Herreras O, Solís JM, Martín del Río R, Lerma J (1987) Characteristics of CA 1 activation through the hippocampal trisynaptic pathway in the unanaesthetized rat. Brain Res 413:75-86.

Herreras O, Menéndez N, Herranz AS, Solís, JM and Martín del Río $R$ (1989) Synaptic transmission at the Schaffer-CAl synapse is blocked by 6,7-dinitro-quinoxaline-2,3-dione. An in vivo brain dialysis study in the rat. Neurosci Lett 99:119-124.

Higashida H, Mitarai G, Watanabe S (1974) A comparative study of membrane potential changes in neurons and neuroglial cells during spreading depression in the rabbit. Brain Res 65:411-425.

Ichijo M, Ochs S (1970) Spreading depression of negative wave of direct cortical response and pyramidal tract responses. Brain Res 23: 41-56.

Jing J, Aitken PA, Somjen GG (1993) Role of calcium channels in spreading depression in rat hippocampal slices. Brain Res 604:251259.

Kraig RP, Nicholson C (1978) Extracellular ionic variations during spreading depression. Neuroscience 3:1045-1059.

Largo C, Somjen GG, Ibarz JM, Martín del Río R, Herreras O (1993) Synchronization ahead of the spreading depression is not operated by Glu-mediated chemical transmission. Eur J Neurosci [Suppl] 6:61.

Leão AAP (1944) Spreading depression of activity in the cerebral cortex. J Neurophysiol 7:359-390.

Lemenkühler A (1990) Spreading depression-Reaktionen an der Hirnrinde: Störungen des Extrazellulären Mikromilieus. Z EEG-EMG 21:1-6.

MacVicar BA, Dudek FE (1981) Electrotonic coupling between pyramidal cells: a direct demonstration in rat hippocampal slices. Science 213:782-785.

Marshall WH (1959) Spreading cortical depression of Leão. Physiol Rev 39:239-279.

Morlock NL, Mori K, Ward AA (1964) A study of single cortical neurons during spreading depression. J Neurophysiol 27:1192-1198.

Muñoz E (1970) Facilitation of cortical cell activity during spreading depression. J Neurobiol 2:47-60.

Mutch WAC, Hansen AJ (1984) Extracellular pH changes during spreading depression and cerebral ischemia: mechanisms of brain $\mathrm{pH}$ regulation. J Cereb Blood Flow Mctab 4:17-27.

Nellgärd B, Wieloch T (1992) NMDA-receptor blockers but not NBQX, an AMPA-receptor antagonist, inhibit spreading depression in the rat brain. Acta Physiol Scand 146:497-503.

Nicholson C (1984) Comparative neurophysiology of spreading depression in the cerebellum. An Acad Bras Cienc 56:481-494.

Rosenblueth A, García-Ramos J (1966) Some phenomena usually associated with spreading depression. Acta Physiol Latinoam 16:141179. 
Sherman A, Rinzel J (1992) Rhythmogenic effects of weak electrotonic coupling in neuronal models. Proc Natl Acad Sci USA 89:2471-2474.

Siesjö BK, Bengtsson F (1989) Calcium fluxes, calcium antagonists, and calcium related pathology in brain ischemia, hypoglycaemia, and spreading depression: a unifying hypothesis. J Cereb Blood Flow Metab 9:127-140.

Snow RW, Dudek FE (1984) Electrical fields directly contribute to action potential synchronization during convulsant-induced epileptiform bursts. Brain Res 323:114-118.

Somjen GG (1981) The why and the how of measuring the activity of ions in extracellular fluid of spinal cord and cerebral cortex. In: The application of ion-selective electrodes (Zeuthen T, ed), pp 175194. Amsterdam: Elsevier.

Somjen GG, Aitken PG (1984) The ionic and metabolic responses associated with neuronal depression of Leão's type in cerebral cortex and in hippocampal formation. An Acad Bras Cienc 56:495-504.

Somjen GG, Aitken PG, Giacchino JL, McNamara JO (1985) Sustained potential shifts and paroxysmal discharges in hippocampal formation. J Neurophysiol 53:1079-1097.

Somjen GG, Aitken PG, Czéh GL, Herreras O, Jing J, Young JN (1992) The mechanisms of spreading depression: a review of recent findings, and a hypothesis. Can J Physiol Pharmacol [Suppl] 70:S248-S254.

Spira ME, Bennett MVL (1972) Synaptic control of electrotonic coupling between neurons. Brain Res 37:294-300.
Stone J, Freeman JA (1971) Synaptic organization of the pigeon's optic tectum: a Golgi and current source-density analysis. Brain Res 27:203-221.

Sugaya E, Takato M, Noda Y (1975) Neuronal and glial activity during spreading depression in cerebral cortex of cat. J Neurophysiol 38 : 822-841.

Szerb JC (1991) Glutamate release and spreading depression in the fascia dentata in response to microdialysis with high $\mathrm{K}^{+}$: role of glia. Brain Res 542:259-265.

Taylor CP, Dudek FE (1982a) Synchronous afterdischarges in rat hippocampal slices without active chemical synapses. Science 218:810 812.

Taylor CP, Dudek FE (1982b) A physiological test for electrotonic coupling between pyramidal cells in rat hippocampal slices. Brain Res 235:351-357.

Tobiasz C, Nicholson C (1982) Tetrodotoxin resistant propagation and extracellular sodium changes during spreading depression in rat cerebellum. Brain Res 241:329-333.

Van Harreveld A (1959) Compounds in brain extracts causing spreading depression of cerebral cortical activity and contraction of crustacean muscle. J Neurochem 3:300-315. 